\title{
Model-Free, Regularized, Fast, and Robust Analytical Orientation Distribution Function Estimation
}

\author{
Jian Cheng ${ }^{1,2}$, Aurobrata Ghosh ${ }^{2}$, Rachid Deriche ${ }^{2}$, and Tianzi Jiang ${ }^{1}$ \\ ${ }^{1}$ Center for Computational Medicine, LIAMA, Institute of Automation, \\ Chinese Academy of Sciences, China \\ 2 Athena Project Team, INRIA Sophia Antipolis - Méditerranée, France \\ jiancheng@nlpr.ia.ac.cn
}

\begin{abstract}
High Angular Resolution Imaging (HARDI) can better explore the complex micro-structure of white matter compared to Diffusion Tensor Imaging (DTI). Orientation Distribution Function (ODF) in HARDI is used to describe the probability of the fiber direction. There are two type definitions of the ODF, which were respectively proposed in Q-Ball Imaging (QBI) and Diffusion Spectrum Imaging (DSI). Some analytical reconstructions methods have been proposed to estimate these two type of ODFs from single shell HARDI data. However they all have some assumptions and intrinsic modeling errors. In this article, we propose, almost without any assumption, a uniform analytical method to estimate these two ODFs from DWI signals in q space, which is based on Spherical Polar Fourier Expression (SPFE) of signals. The solution is analytical and is a linear transformation from the q-space signal to the ODF represented by Spherical Harmonics (SH). It can naturally combines the DWI signals in different Q-shells. Moreover It can avoid the intrinsic Funk-Radon Transform (FRT) blurring error in QBI and it does not need any assumption of the signals, such as the multiple tensor model and mono/multi-exponential decay. We validate our method using synthetic data, phantom data and real data. Our method works well in all experiments, especially for the data with low SNR, low anisotropy and non-exponential decay.
\end{abstract}

\section{Introduction}

High Angular Resolution Diffusion Imaging (HARDI) is used to probe non-Gaussian diffusion which represents more intricate micro-structure in the tissue. Orientation Distribution Function (ODF) [1/2] was proposed to describe the fiber directions. There are two type of ODFs. One is denoted as $\Phi_{t}$, proposed using radial projection by Tuch in QBI [1]. Another one is denoted as $\Phi_{w}$, proposed as the marginal probability of the Ensemble Average Propagator (EAP) $P(R \mathbf{r})$ by Wedeen in DSI [2]. $\Phi_{t}$ need to be normalized and $Z$ is the normalization factor. While $\Phi_{w}$ is naturally normalized.

$$
\Phi_{t}(\mathbf{r})=\frac{1}{Z} \int_{0}^{\infty} P(R \mathbf{r}) d R \quad \Phi_{w}(\mathbf{r})=\int_{0}^{\infty} P(R \mathbf{r}) R^{2} d R=\frac{1}{2} \int_{-\infty}^{\infty} P(R \mathbf{r}) R^{2} d R
$$

where $\mathbf{R}=R \mathbf{r}$ is the displacement in $3 \mathrm{D}$ space. It has been shown that $\Phi_{w}$ is more sharper than $\left.\Phi_{t}[2] 34\right]$, which means $\Phi_{w}$ is more discriminative for fiber detection. 
Historically, Funk-Radon Transform (FRT) was used in QBI to estimate $\Phi_{t}$ numerically [1] or analytically [5]. However, the intrinsic blurring effect of FRT can bring some errors [1]. $\Phi_{w}$ was firstly proposed in DSI and was estimated from numerical radial integral after the numerical Fourier Transform of the signals [2]. Most recently, several similar analytical reconstruction methods were proposed separately to estimate $\Phi_{w}$ from single shell HARDI data [6/3 4]. Elegant analytical solutions were found [6/3 4] based on the mono-exponential decay assumption [7] which gives the full information about $E(\mathbf{q})$ in the whole $3 \mathrm{D} \mathbf{q}$-space from the $E\left(\mathbf{q}_{0}\right)$ only in a single shell. The approximated EAP $\tilde{P}(R \mathbf{r})$ actually is the true EAP $P(R \mathbf{r})$ convolved by the Fourier transform of the function $E(q, \mathbf{u})^{q^{2} / q_{0}^{2}} E(q, \mathbf{u})^{-1}[7]$, where $\mathbf{q}=q \mathbf{u}, q=\|\mathbf{q}\|$. It was shown surprisingly that the estimated $\tilde{P}(R \mathbf{r})$ and $\tilde{\Phi}_{w}$ are sharper than the real $P(R \mathbf{r})$ and $\left.\Phi_{w}[6] 3\right]$ in the synthetic data generated from mixture tensor model. However, since this surprising results come from the intrinsic modeling error from the unrealistic kernel smooth, it is still not clear if the methods based on that assumption can work well in the complex real data with non-exponential decay, low anisotropy and low SNR. Similarly with [7], the authors in [3] extended mono-exponential model to multi-exponential model so that it can reduce the modeling error and work for the data in multiple shells. However, it is impractical because a nonlinear fitting is needed for every direction [7], suffering from limited samples, local minima, computational complexity, and an analytic solution exists only when three $b$ values satisfy an arithmetic process.

$$
\begin{array}{cc}
E(\mathbf{q})=\sum_{n=0}^{N} \sum_{l=0}^{L} \sum_{m=-l}^{l} a_{n, l, m} R_{n}(\|\mathbf{q}\|) Y_{l}^{m}(\mathbf{u}) & B_{n, l, m}(\mathbf{q})=R_{n}(\|\mathbf{q}\|) Y_{l}^{m}(\mathbf{u}) \\
R_{n}(\|\mathbf{q}\|)=\kappa_{n}(\zeta) \exp \left(-\frac{\|\mathbf{q}\|^{2}}{2 \zeta}\right) L_{n}^{1 / 2}\left(\frac{\|\mathbf{q}\|^{2}}{\zeta}\right) & \kappa_{n}(\zeta)=\left[\frac{2}{\zeta^{3 / 2}} \frac{n !}{\Gamma(n+3 / 2)}\right]^{1 / 2}
\end{array}
$$

In [8], the Spherical Polar Fourier Expression (SPFE) was proposed to sparsely represent $E(\mathbf{q})$. See formulae (2), (3), where $Y_{l}^{m}(\mathbf{u})$ is the $l$ order $m$ degree Spherical Harmonic (SH) basis and $R_{n}(q)$ is the Gaussian-Laguerre polynomial basis. Since $B_{n, l, m}(\mathbf{q})$ is the orthonormal basis in $R^{3}$, any type of $E(\mathbf{q})$ could be represented by a linear combination of $\left\{B_{n, l, m}\right\}$. After the coefficients $\left\{a_{n, l, m}\right\}$ of the signal are estimated from a least square fit or a nonlinear robust estimation [8], $\Phi_{t}$ could be calculated through an inner product of the coefficients $a_{n, l, m}$ and a kernel $b_{n, l, m}$. The problem in [8] is that $b_{n, l, m}$ needs to be calculated numerically from FFT for every direction or calculated for one direction then rotated by Wigner rotation matrix for other directions. That is inefficient and can bring some numerical error, especially for these kernels which have some delta functions inside, e.g. the kernels for $\Phi_{t}$ and $\Phi_{w}$. And it can not provide an elegant analytical parametrized result like analytical QBI [5].

In this paper, instead of adding strong assumptions for single shell data in [634] and numerical solution using FFT and Wigner matrix in [8], we propose a uniform analytical estimation method for $\Phi_{t}$ and $\Phi_{w}$ based on SPFE, which includes two linear transformations from the coefficients $\left\{a_{n, l, m}\right\}$ of $E(\mathbf{q})$ to the coefficients $\left\{c_{l, m}^{t}\right\}$ of $\Phi_{t}$ and $\left\{c_{l, m}^{w}\right\}$ of $\Phi_{w}$ represented by SHs. First we deduce the transformations for $\Phi_{t}$ and $\Phi_{w}$. Next, we perform the method in some non-exponential synthetic data and a challenging phantom data. At last we test our methods in a real monkey data with several $b$ values. 


\section{Analytical ODF Estimation Based On SPF}

It has been shown that the line integral of $P(R \mathbf{r})$ in $\mathbf{R}$-space in (1) is equivalent to the integral in the plane in $\mathbf{q}$-space which is orthogonal to the line in $\mathbf{R}$-space [346]. See formula (4), where $\triangle_{b}$ is the Laplace-Beltrami operator. Our contribution is to deduce the elegant analytical solution for data in multiple shells based on these previous studies in [3468]. Our analytical estimation methods almost do not need any assumption about the signal. The only assumption we need is that the signal $E(\mathbf{q})$ can be sparsely represented by SPF in formula (2), which has been validated in [8].

$$
\Phi_{t}(\mathbf{r})=\frac{1}{Z} \iint_{\Pi_{\mathbf{r}}} E(\mathbf{q}) q \delta\left(\mathbf{r}^{T} \mathbf{u}\right) d q d \mathbf{u} \quad \Phi_{w}(\mathbf{r})=\frac{1}{4 \pi}-\frac{1}{8 \pi^{2}} \iint_{\Pi_{\mathbf{r}}} \frac{\Delta_{b} E(\mathbf{q})}{q} \delta\left(\mathbf{r}^{T} \mathbf{u}\right) d q d \mathbf{u}
$$

\subsection{Estimation of $\Phi_{t}$}

Put the formula (2) into (4), we can easily get the solution.

$$
\begin{aligned}
\Phi_{t}(\mathbf{r}) & =\frac{1}{Z} \iint_{\Pi_{\mathbf{r}}} \sum_{n=0}^{N} \sum_{l=0}^{L} \sum_{m=-l}^{l} a_{n, l, m} R_{n}(q) Y_{l}^{m}(\mathbf{u}) q \delta\left(\mathbf{r}^{T} \mathbf{u}\right) d q d \mathbf{u} \\
& =\frac{1}{Z} \sum_{n=0}^{N} \sum_{l=0}^{L} \sum_{m=-l}^{l} a_{n, l, m}\left(\int_{0}^{2 \pi} Y_{l}^{m}(\mathbf{u}) \delta\left(\mathbf{r}^{T} \mathbf{u}\right) d \mathbf{u}\right)\left(\int_{0}^{\infty} R_{n}(q) q d q\right) \\
& =\frac{1}{Z} \sum_{n=0}^{N} \sum_{l=0}^{L} \sum_{m=-l}^{l} a_{n, l, m}\left(2 \pi P_{l}(0) Y_{l}^{m}(\mathbf{r})\right)\left(\frac{\kappa_{n}(\zeta) \zeta}{2} \int_{0}^{\infty} \exp \left(-\frac{x}{2}\right) L_{n}^{1 / 2}(x) d x\right) \\
& =\frac{2 \pi \zeta}{Z} \sum_{l=0}^{L} \sum_{m=-l}^{l}\left(\sum_{n=0}^{N} \sum_{i=0}^{n} \kappa_{n}(\zeta)\left(\begin{array}{c}
i-0.5 \\
i
\end{array}\right)(-1)^{n-i} P_{l}(0) a_{n, l, m}\right) Y_{l}^{m}(\mathbf{r})
\end{aligned}
$$

where $P_{l}(0)$ is the Legendre polynomial of order $l$ at 0 . We get (5) because SH is the eigenfunction of the FRT [5]. From (5) to (6), we use the property of Laguerre polynomial [9]. Thus here we have a linear transformation from the coefficients $a_{n, l, m}$ of $E(\mathbf{q})$ to the coefficients $c_{l, m}^{t}=\sum_{n=0}^{N} \sum_{i=0}^{n} \kappa_{n}(\zeta)\left(\begin{array}{c}i-0.5 \\ i\end{array}\right)(-1)^{n-i} P_{l}(0) a_{n, l, m}$. Please note that the author in [10] gave a solution for $\Phi_{t}$ in page 122. Unfortunately, the integrand there was wrong because of wrong volume element. Here we give the right analytical formulae.

We also give the result of the integral in a given disk $\Pi_{(\mathbf{r}, C)}$ whose radius is $C$. In the formula (4), the integral $\Phi_{t}(\mathbf{r})$ gives the same weight for $E(\mathbf{q})$ with large $q$ and for $E(\mathbf{q})$ with small $q$. However, if we just have several b values, the error of estimated signal $\tilde{E}(\mathbf{q})$ will be small if $q$ is between these b values and will be large if $q$ is large than all $\mathrm{b}$ values. Thus if an approximate $C$ is given, the disk integral $\Phi_{t}(\mathbf{r}, C)$ may have better angular resolution than $\Phi_{t}(\mathbf{r})$ [4]. Considering $L_{n}^{1 / 2}(x)=\sum_{i=0}^{n} l_{n}^{i} x^{i}, l_{n}^{i}=(-1)^{i}\left(\begin{array}{c}n+0.5 \\ n-i\end{array}\right) \frac{1}{i !}$, and the lower incomplete gamma function $\gamma(i, x)=\int_{0}^{x} t^{i-1} \exp (-t) d t$, we have

$$
\begin{aligned}
\Phi_{t}(\mathbf{r}, C) & =\frac{2 \pi \zeta}{Z} \sum_{n=0}^{N} \sum_{l=0}^{L} \sum_{m=-l}^{l} a_{n, l, m}\left(P_{l}(0) Y_{l}^{m}(\mathbf{r})\right)\left(\frac{\kappa_{n}(\zeta)}{2} \int_{0}^{C^{2} / \zeta} \exp \left(-\frac{x}{2}\right) L_{n}^{1 / 2}(x) d x\right) \\
& =\frac{2 \pi \zeta}{Z} \sum_{l=0}^{L} \sum_{m=-l}^{l}\left(\sum_{n=0}^{N} \sum_{i=0}^{n} \kappa_{n}(\zeta)\left(\begin{array}{c}
n+0.5 \\
n-i
\end{array}\right) \frac{(-2)^{i}}{i !} \gamma\left(i+1,0.5 C^{2} / \zeta\right) P_{l}(0) a_{n, l, m}\right) Y_{l}^{m}(\mathbf{r})
\end{aligned}
$$




\subsection{Estimation of $\Phi_{w}$}

Similarly, put the formula (2) into (4) we can get the analytical expression for $\Phi_{w}$.

$$
\Phi_{w}(\mathbf{r})=\frac{1}{4 \pi}-\frac{1}{8 \pi^{2}} \sum_{n=0}^{N} \sum_{l=0}^{L} \sum_{m=-l}^{l} a_{n, l, m}\left(\int_{0}^{2 \pi} \triangle_{b} Y_{l}^{m}(\mathbf{u}) \delta\left(\mathbf{r}^{T} \mathbf{u}\right) d \mathbf{u}\right)\left(\int_{0}^{\infty} \frac{R_{n}(q)}{q} d q\right)
$$

However, we can not solve it just like what we did for $\Phi_{t}$, because the division by $q$ introduces a pole. It is a little hard to find the analytical solution for $\Phi_{w}$. And the author in [10]8] did not give any solution for that. We solve this problem by considering $E(0)=1$, which is a true fact for any DWI data. That means, for our basis, the identity $E(0)=\sum_{n, l, m} a_{n, l, m} R_{n}(0) Y_{l}^{m}(\mathbf{u})=\sum_{n, l, m} a_{n, l, m} \kappa_{n}(\zeta) Y_{l}^{m}(\mathbf{u})=1$ holds for any $\mathbf{u} \in S^{2}$. Also keep in mind that a constant addition inside $\triangle_{b}$ does not change the final result. First we consider the integral inside a given disk $\Pi_{(\mathbf{r}, C)}$, then we have

$$
\begin{aligned}
\Phi_{w}(\mathbf{r}, C) & =\frac{1}{4 \pi}-\frac{1}{8 \pi^{2}} \iint_{\Pi_{(\mathbf{r}, C)}} \frac{\Delta_{b}(E(\mathbf{q})-E(0))}{q} \delta\left(\mathbf{r}^{T} \mathbf{u}\right) d q d \mathbf{u} \\
& =\frac{1}{4 \pi}-\frac{1}{8 \pi^{2}} \sum_{n=0}^{N} \sum_{l=0}^{L} \sum_{m=-l}^{l} a_{n, l, m}\left(\int_{0}^{2 \pi} \triangle_{b}\left(\int_{0}^{C} \frac{R_{n}(q)-R_{n}(0)}{q} d q\right) Y_{l}^{m}(\mathbf{u}) \delta\left(\mathbf{r}^{T} \mathbf{u}\right)\right) d \mathbf{u} \\
& =\frac{1}{4 \pi}-\frac{1}{8 \pi^{2}} \sum_{n=0}^{N} \sum_{l=0}^{L} \sum_{m=-l}^{l} a_{n, l, m} \kappa_{n}(\zeta)\left(\int_{0}^{2 \pi} \triangle_{b} I_{n}(C) Y_{l}^{m}(\mathbf{r}) \delta\left(\mathbf{r}^{T} \mathbf{u}\right) d \mathbf{u}\right)
\end{aligned}
$$

Now there is no pole! For $I_{n}(C)$ we have

$$
\begin{aligned}
I_{n}(C) & =\int_{0}^{C} \frac{R_{n}(q)-R_{n}(0)}{\kappa_{n}(\zeta) q} d q \\
& =\frac{1}{2} \int_{0}^{C^{2} / \zeta}\left(\frac{\exp (-x / 2)-1}{x}+\sum_{i=1}^{n} l_{n}^{i} x^{i-1} \exp \left(-\frac{x}{2}\right)\right) d x \\
& =\underbrace{0.5\left(-\gamma-E_{1}\left(0.5 C^{2} / \zeta\right)-\log \left(0.5 C^{2} / \zeta\right)\right)}_{I_{n}^{1}(C)}+\underbrace{0.5 \sum_{i=1}^{n} l_{n}^{i} 2^{i} \gamma\left(i, 0.5 C^{2} / \zeta\right)}_{I_{n}^{2}(C)}
\end{aligned}
$$

where $\gamma \simeq 0.5772$ is the Euler-Mascheroni constant, $E_{1}(x)=\int_{x}^{\infty} \frac{\exp (-t)}{t} d t$ is the exponential integral. Although there are two parts in $I_{n}(C)$ and $I_{n}^{1}(C)$ tends to infinity, it actually has no contribution for $\Phi_{w}(\mathbf{r}, C)$, because $\sum_{n, l, m} a_{n, l, m} \kappa_{n}(\zeta) Y_{l}^{m}(\mathbf{u}) I_{n}^{1}(C)=I_{n}^{1}(C)$ is a constant inside $\Delta_{b}$. Then considering $Y_{l}^{m}(\mathbf{u})$ is the eigenfunction of FRT and $\triangle_{b}$, we have the analytical result for $\Phi_{w}(\mathbf{r}, C)$ and $\Phi_{w}(\mathbf{r})=\lim _{C \rightarrow \infty} \Phi_{w}(\mathbf{r}, C), c_{l, m}^{w}=\lim _{C \rightarrow \infty} c_{l, m}^{w}(C)$

$$
\begin{gathered}
\Phi_{w}(\mathbf{r}, C)=\frac{1}{4 \pi}-\frac{1}{8 \pi} \sum_{n, l, m} a_{n, l, m} \kappa_{n}(\zeta) \sum_{i=1}^{n} l_{n}^{i} 2^{i} \gamma\left(i, 0.5 C^{2} / \zeta\right) P_{l}(0)(-l)(l+1) Y_{l}^{m}(\mathbf{r}) \\
c_{l, m}^{w}(C)=\frac{1}{\sqrt{4 \pi}} \delta(l) \delta(m)-\frac{1}{8 \pi} \sum_{n=1}^{N} \sum_{i=1}^{n}(-1)^{i} \kappa_{n}(\zeta)\left(\begin{array}{c}
n+0.5 \\
n-i
\end{array}\right) \frac{2_{i !}^{i}}{i !}\left(i, 0.5 C^{2} / \zeta\right) P_{l}(0)(-l)(l+1) a_{n, l, m}
\end{gathered}
$$




$$
c_{l, m}^{w}=\frac{1}{\sqrt{4 \pi}} \delta(l) \delta(m)-\frac{1}{8 \pi} \sum_{n=1}^{N} \sum_{i=1}^{n}(-1)^{i} \kappa_{n}(\zeta)\left(\begin{array}{c}
n+0.5 \\
n-i
\end{array}\right) \frac{2^{i}}{i} P_{l}(0)(-l)(l+1) a_{n, l, m}
$$

Now we have two estimations for the true $\Phi_{w}$. One is the integral in the whole plane, which is similar with [3], and another one is the integral in a given disk, which is similar with [4]. However, the mono-exponential decay model was assumed during the disk in [4], and in the whole plane in [3], so that the integral for the radial integral could be approximated just using the data in the q-circle. While our method does not need any assumption on the data and it can handle the data in different q-shells. Also please note three important points in the formulae above. First, we get the the $E_{1}(x)$ in the derivation process, but it is negligible. While in [4], it is indispensable. Second, the formulae (15), (16) tell us that at least order 1 of $R_{n}$ is needed to represent an anisotropic ODF. That is true because if we just use the radial basis of order zero, it is easily seen that the estimated signal is just an isotropic one, which means the estimated ODF is isotropic. Thus we need to use at least two shells to get a reasonable results, although our methods can be performed in single shell data. Third, since in (4) $1 / q$ gives small weight for $E(\mathbf{q})$ with large $q$ and large weight for $E(\mathbf{q})$ with small $q$, that means the error in large $q$ may be negligible! Thus $\Phi_{w}$ is more robust to estimation error of $E(\mathbf{q})$. How to choose an approximate $C$ for $\Phi_{w}(\mathbf{r}, C)$ and $\Phi_{t}(\mathbf{r}, C)$ is still an open question out of the scope of this paper. And since the improvement of $\Phi_{w}(C)$ in [4] over $\Phi_{w}$ in [3] is very subtle [4], here we just consider the $\Phi_{w}$ and $\Phi_{t}$, not $\Phi_{w}(C), \Phi_{t}(C)$.

\subsection{Implementation of Methods}

The Implementation includes two steps. The first step is to estimate coefficients $\left\{a_{n, l, m}\right\}$ from the observed signals $\left\{E\left(\mathbf{q}_{i}\right)\right\}$. The second step is the uniform linear analytical solution proposed above from $\left\{a_{n, l, m}\right\}$ to $\left\{c_{l, m}^{t}\right\}$ and $\left\{c_{l, m}^{w}\right\}$, which is actually independent of the first step. The whole estimation error is just from the first step, since the second step is analytical and compact. The authors in [8] suggested two methods to estimate $\left\{a_{n, l, m}\right\}$, a linear least square (LS) fitting with regularization in the radial and spherical parts, and a non-linear PDE based optimization process, which considers the Rician noise. Here we choose the LS method in the first step since it is more faster. We suggest that the Rician correction could be performed directly on the DWI data as a preprocessing step [11|12], although in our experiments to perform an appropriate comparison of methods we did not do any Rician correction. For LS estimation, let's denote the signal vector by $E=\left[E\left(\mathbf{q}_{i}\right)\right]_{S \times 1}$, the coefficient vector by $A=\left[a_{n, l, m}\right]_{0.5(L+1)(L+2)(N+1) \times 1}$, the basis matrix by $M=\left[R_{n}(\mathbf{q}) Y_{l}^{m}(\mathbf{u})\right]_{S \times 0.5(L+1)(L+2)(N+1)}$, and the spherical and radial regularization diagonal matrices respectively by $L=[l(l+1)]$ and $N=[n(n+1)]$, where $\lambda_{l}$ and $\lambda_{n}$ are the regularization terms for spherical and radial parts. Then $A=$ $\left(M^{T} M+\lambda_{l} L^{T} L+\lambda_{n} N^{T} N\right) M^{T} E$. For the second step, the linear transformations in (6), (7), (15), (16) could be also implemented as a matrix multiplication. Thus the whole process is just a linear matrix multiplication on the data vector $E$. Similarly with analytical Q-ball in [5], the matrix is independent of $E$ and needs to be calculated only once for the whole data set. It makes our method extremely fast.

There are two important points to consider in the implementation. The first one is about $E(0)$. If we have a data set with several b values, $b_{1}, b_{2} \ldots, b_{N}$, we actually use 
$N+1 \mathrm{~b}$ values, considering $E(0)=1$ for any $\mathbf{u} \in S^{2}$, which makes our estimation more reasonable and accurate. Otherwise, there is no warranty for the estimated signal $\tilde{E}(0)=1$. For the single shell HARDI data, considering $b=0$ can let us have 2 shells, which will improve the results. The second one is how to determine the parameter $\zeta$ in (3). The authors in [8] proposed a strategy for it, which is dependent on the radial truncation order $N$. However, we think the parameter should be just dependent on the signal, not on the basis order. Considering $E(\mathbf{q})=\exp \left(-4 \pi^{2} \tau q^{2} D\right), b=4 \pi^{2} \tau q^{2}$, and a typical diffusion coefficient of $D=0.7 \times 10^{-3} \mathrm{~mm}^{2} / \mathrm{s}$, a typical b-value $b=3000 \mathrm{~s} / \mathrm{mm}^{2}$, we set $\zeta=\frac{1}{8 \pi^{2} \tau \times 0.7 \times 10^{-3}}$. If $4 \pi^{2} \tau=1$, then $\zeta$ is about 700. In our experiments we always set $\zeta=700$.

\section{Results on Synthetic, Phantom and Real Data}

Synthetic data. Gaussian mixture model $S\left(\mathbf{q}_{s}\right)=\sum_{i=1}^{M} p_{i} G_{i}\left(\mathbf{q}_{s}\right), G_{i}\left(\mathbf{q}_{s}\right)=$ $\exp \left(-q_{s}^{2} \mathbf{u}_{s}^{T} \mathbf{D}_{i} \mathbf{u}_{s}\right)$ has been used widely to generate synthetic data [1 563]4]. However, that could bias the results in favor of those methods assuming a model based on Gaussian mixture or mono/multi-exponential decay. Here we choose both Gaussian mixture and non-Gaussian mixture to validate our methods. We set $S(\mathbf{q})=\sum_{i=1}^{M} p_{i} f_{i}(\mathbf{q})$, $f(\mathbf{q})=G(\mathbf{q})$ for a Gaussian mixture model and $f(\mathbf{q})=0.5 G(\mathbf{q})+0.5 T(\mathbf{q}), T(\mathbf{q})=$ $\exp \left(-\sqrt{2 q^{2} \mathbf{u}^{T} \mathbf{D u}}\right)$ for a non-Gaussian mixture model. It could be proved that the $\Phi_{t}$ and $\Phi_{w}$ of $T(\mathbf{q})$ are the same as the ODFs of $G(\mathbf{q})$, although they have different EAPs [9]. Thus we have the analytical ground truth of ODFs. We set the eigenvalues of $\mathbf{D}$ as $[0.3,0.3,1.7] \times 10^{-3} \mathrm{~mm}^{2} / \mathrm{s}$. and use the same way in [5] to add Rician noise with $S N R=1 / \sigma$, which is defined as the ratio of maximal signal intensity of $S(0)=1$ to the standard deviation $\sigma$ of complex Gaussian noise. We test the methods with $S N R=10$ in four configurations of ODF: one fiber or two orthogonal fibers with Gaussian model or non-Gaussian model. For each configuration, data in 4 shells $\left(\mathrm{b}=500,1000,2000,3000 \mathrm{~s} / \mathrm{mm}^{2}\right)$ were generated. For the single shell methods, e.g. $\Phi_{t}$ estimation in [5] and $\Phi_{w}$ estimation in [3], 4 order SH with $\lambda=0.006$ was chosen. For the proposed methods, we use all data from four shells and chose $N=2, L=4$, $\lambda_{n}=5 e-8, \lambda_{l}=1 e-7, \zeta=700$. We recorded the percentage of correct number of detected ODF maximum and the mean of angular error over 1000 trails [5]. See table 1. The experiments showed: 1) Normally, the $\Phi_{t}$ is more robust to Rician noise than $\Phi_{w}$, although $\Phi_{w}$ is more sharper and has better angular resolution. It is the similar conclusion in [4]. 2) our methods for $\Phi_{t}$ and $\Phi_{t}$ both got better performance than the methods for single shell data in [5]3]. 3) the method in [3] will get worse results for the data with non-exponential decay or much noise.

Phantom data. We performed our methods in a public phantom data with 3 shells, where b-value is $650,1500,2000 \mathrm{~s} / \mathrm{mm}^{2}$ respectively. This data has been used in the fiber cup contest in MICCAI 2009 to evaluate tracking methods [13]. The anisotropy of this data is very low, which makes it hard to detect the fibers. We believe that it is complex enough to evaluate different reconstruction methods and tracking methods. We compare our reconstruction method using 3 shells with the method in [3] using one shell $(b=2000)$, since the result of $b=2000$ is better than the results of $b=650$ and 1500 for the method in [3]. For our method, we choose $L=4, \lambda_{l}=5 e-8$ in the 
Table 1. Each column shows the percentage of correct number of detected ODF maximum and the mean of angular errors under an given ODF configuration. The left part and the right part in each column are respectively for the estimation methods for $\Phi_{t}$ and $\Phi_{w}$. The first four rows recorded the performance of previous works on single shell data in [5] and in [3] with 81 gradient directions on the hemisphere. The last row shows the results of our methods using 4 shells.

\begin{tabular}{|l||l|l|l|l|l|}
\hline b value $\mid$ 1 fiber (Gaussian) & 1 fiber (non-Gaussian) & $\mid 2$ fiber (Gaussian) & $\mid 2$ fiber (non-Gaussian) \\
\hline \begin{tabular}{l||l|l|l}
500 \\
1000
\end{tabular} & $\left(100 \%, 4.8^{\circ}\right)\left(91.8 \%, 7.0^{\circ}\right)$ & $\left(100 \%, 5.2^{\circ}\right)\left(79.6 \%, 7.6^{\circ}\right)$ & $\left(71.0 \%, 17.5^{\circ}\right)\left(57.4 \%, 19.5^{\circ}\right)$ & $\left(74.6 \%, 16.0^{\circ}\right)\left(43.6 \%, 18.7^{\circ}\right)$ \\
2000 & $\left(100 \%, 3.1^{\circ}\right)\left(99.9 \%, 4.6^{\circ}\right)$ & $\left(100 \%, 4.0^{\circ}\right)\left(95.6 \%, 6.2^{\circ}\right)$ & $\left(88.6 \%, 9.5^{\circ}\right)\left(80.9 \%, 10.5^{\circ}\right)$ & $\left(87.7 \%, 11.4^{\circ}\right)\left(63.0 \%, 12.1^{\circ}\right)$ \\
3000 & $\left(100 \%, 2.6^{\circ}\right)\left(99.9 \%, 3.5^{\circ}\right)$ & $\left(100 \%, 3.7^{\circ}\right)\left(90.1 \%, 4.8^{\circ}\right)$ & $\left(98.1 \%, 6.9^{\circ}\right)\left(87.5 \%, 7.0^{\circ}\right)$ & $\left(93.1 \%, 9.5^{\circ}\right)\left(60.7 \%, 9.9^{\circ}\right)$ \\
4 shells & $\left(100 \%, 3.1^{\circ}\right)\left(87.6 \%, 4.0^{\circ}\right)$ & $\left(99.4 \%, 4.6^{\circ}\right)\left(52.0 \%, 5.2^{\circ}\right)$ & $\left(95.6 \%, 7.7^{\circ}\right)\left(56.0 \%, 7.9^{\circ}\right)$ & $\left(85.3 \%, 11.2^{\circ}\right)\left(40.4 \%, 12.1^{\circ}\right)$ \\
$\left(100 \%, 2.0^{\circ}\right)\left(100 \%, 2.5^{\circ}\right)$ & $\left(100 \%, 3.1^{\circ}\right)\left(98.5 \%, 3.4^{\circ}\right)$ & $\left(99.8 \%, 4.5^{\circ}\right)\left(94.7 \%, 5.7^{\circ}\right)$ & $\left(98.5 \%, 5.5^{\circ}\right)\left(78.5 \%, 7.5^{\circ}\right)$ \\
\hline
\end{tabular}

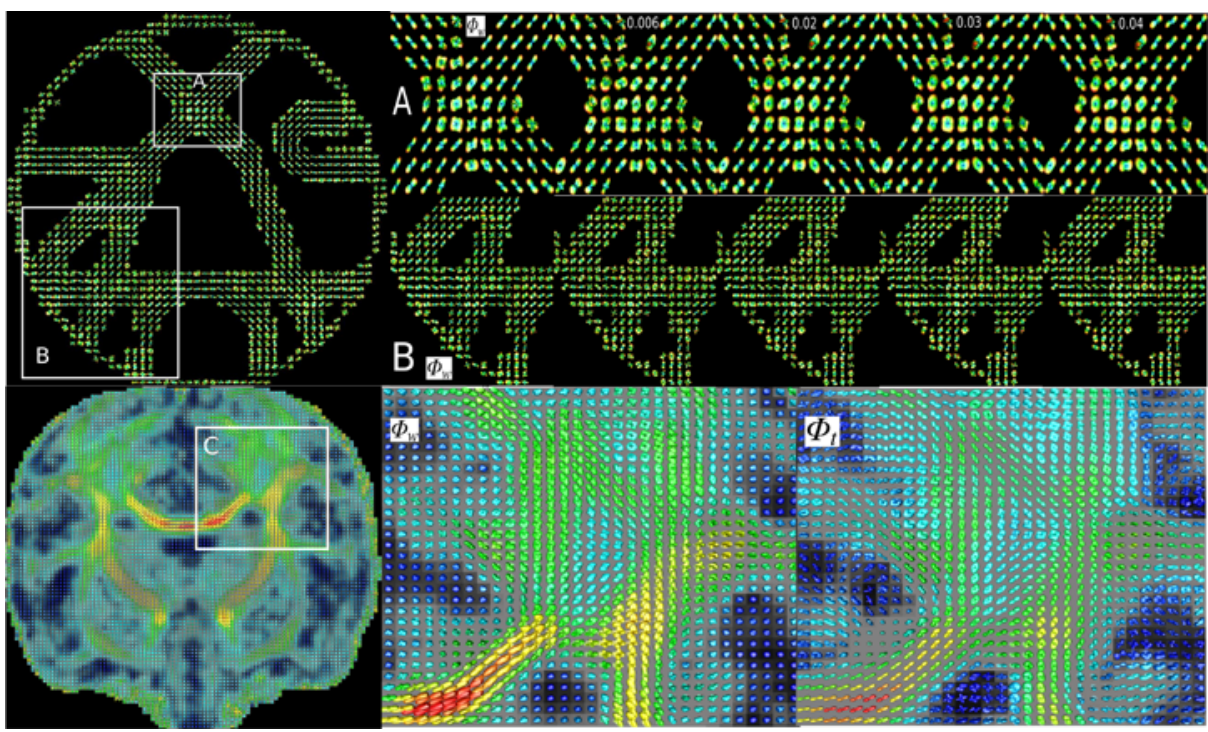

Fig. 1. First row: phantom data, from left to right: whole view of $\Phi_{w}$ from our method, $\Phi_{w}$ in region A and B from our method, method in [3] with $\lambda=0.006,0.02,0.03,0.04$; Second row: real data result from our method, from left to right: whole view of $\Phi_{w}, \Phi_{w}$ and $\Phi_{t}$ in region C

spherical part and $N=1, \lambda_{n}=1 e-9$ in the radial part [8]. To perform a fair comparison, we choose $L=4$ and tune the Laplacian regularization term $\lambda$ from 0.006 (suggested in [53]) to $0.02,0.03$ and 0.04 for the method in [3]. Two crossing areas were chosen for visualization using min-max normalization [1]. The results were shown in Fig. 1. It shows that the method using 3 shells is better. The bad performance of the method in [3] probably comes from the error of the mono-exponential assumption.

Real data. We perform our method in a real monkey data with 3 shells $(b=500,1500$, $3000 \mathrm{~s} / \mathrm{mm}^{2}$ ), 30 directions at each b value, TE/TR/matrix $=120 \mathrm{~ms} / 6000 \mathrm{~ms} / 128 \times 128$. We set $L=4, N=2, \lambda_{l}=5 e-8, \lambda_{n}=1 e-9$ and show, in Fig. 1, the results of $\Phi_{t}$ 
with min-max normalization and $\Phi_{w}$ without normalization, since $\Phi_{w}$ is sharper than $\Phi_{t}$. The glyphs were colored by GFA calculated from ODF [1].

\section{Conclusion}

We proposed a uniform model-free fast robust analytical ODF reconstruction method based on Spherical Polar Fourier (SPF) expression of the signal in q-space. The coefficients of the two kinds of ODF under SH could be linearly and analytically calculated from the coefficients of the signal under SPF. It is a linear transformation that is independent of the data. This transformation matrix is just calculated only once for a whole data set, which makes the method very fast. Our method can avoid the error from unrealistic assumptions and can naturally combine data from different Q-shells. The results in synthetic data phantom data and real data show that our method can get better results compared with previous single shell HARDI methods in [5] and in [3], especially for the data with low anisotropy, low SNR and non-exponential decay.

Acknowledgment. This work was partly supported by the Natural Science Foundation of China (30730035), the National Key Basic Research and Development Program of China (2007CB512305), the National High Technology Research and Development Program of China (2009AA02Z302), the External Cooperation Program of the Chinese Academy of Sciences (GJHZ200826), the French ANR "Neurological and Psychiatric diseases" NucleiPark and the France-Parkinson Association.

\section{References}

1. Tuch, D.S.: Q-ball imaging. Magnetic Resonance in Medicine 52, 1358-1372 (2004)

2. Wedeen, V.J., Hagmann, P., Tseng, W.Y.I., Reese, T.G., Weisskoff, R.M.: Mapping complex tissue architecture with diffusion spectrum magnetic resonance imaging. Magnetic Resonance In Medicine 54, 1377-1386 (2005)

3. Aganj, I., Lenglet, C., Sapiro, G., Yacoub, E., Ugurbil, K., Harel, N.: Reconstruction of the orientation distribution function in single and multiple shell q-ball imaging within constant solid angle. Magnetic Resonance in Medicine (2009)

4. Tristán-Vega, A., Westin, C.F., Aja-Fernández, S.: A new methodology for the estimation of fiber populations in the white matter of the brain with the Funk-Radon transform. NeuroImage 49, 1301-1315 (2010)

5. Descoteaux, M., Angelino, E., Fitzgibbons, S., Deriche, R.: Regularized, fast and robust analytical q-ball imaging. Magnetic Resonance in Medicine 58, 497-510 (2007)

6. Canales-Rodriguez, E.J., Melie-Garcia, L., Iturria-Medina, Y.: Mathematical description of q-space in spherical coordinates: Exact q-ball imaging. Magnetic Resonance In Medicine 61, 1350-1367 (2009)

7. Özarslan, E., Shepherd, T.M., Vemuri, B.C., Blackband, S.J., Mareci, T.H.: Resolution of complex tissue microarchitecture using the diffusion orientation transform (DOT). NeuroImage 31, 1086-1103 (2006)

8. Assemlal, H.E., Tschumperlé, D., Brun, L.: Efficient and robust computation of PDF features from diffusion MR signal. Medical Image Analysis 13, 715-729 (2009)

9. Gradshteyn, I., Ryzhik, I.: Table of Integrals, Series, and Products. Elsevier, Amsterdam (2007) 
10. Assemlal, H.E.: Diffusion MR image analysis for the estimation of tissues local architecture. $\mathrm{PhD}$ thesis, the University of Caen (2010)

11. Tristán-Vega, A., Aja-Fernández, S.: DWI filtering using joint information for DTI and HARDI. Medical Image Analysis 14, 205-218 (2010)

12. Descoteaux, M., Wiest-Daessle, N., Prima, S., Barillot, C., Deriche, R.: Impact of rician adapted non-local means filtering on HARDI. In: Metaxas, D., Axel, L., Fichtinger, G., Székely, G. (eds.) MICCAI 2008, Part II. LNCS, vol. 5242, pp. 122-130. Springer, Heidelberg (2008)

13. Poupon, C., Rieul, B., Kezele, I., Perrin, M., Poupon, F., Mangin, J.: New diffusion phantoms dedicated to the study and validation of high-angular-resolution diffusion imaging (HARDI) models. Magnetic Resonance In Medicine 60(6), 1276-1283 (2008) 